Research Article

\title{
Preparation and Characterization of Nanoparticulate Drug Delivery System for Naproxen Sodium Using Various Desolvating Agents
}

\author{
Huda Syed, Abbaraju Krishna Sailaja \\ RBVRR Women's College of Pharmacy, Barkatpura, Hyderabad, India. \\ —Corresponding author. E-mail: shailaja1234@rediffmail.com
}

Received: Jun. 21, 2019; Accepted: Aug. 16, 2019; Published: Aug. 16, 2019.

Citation: Huda Syed, Abbaraju Krishna Sailaja, Preparation and Characterization of Nanoparticulate Drug Delivery System for Naproxen Sodium Using Various Desolvating Agents. Nano Biomed. Eng., 2019, I I (3): 254-263.

DOI: 10.5101/nbe.v11i3.p254-263.

\begin{abstract}
Naproxen sodium is a non-steroidal anti-inflammatory agent used for the treatment of autoimmune disorders to relieve pain and inflammation. In the present study, naproxen sodium nanoparticles were prepared by desolvation technique using various desolvating agents (butanol and combinations of acetonitrile and butanol), and two methods (continuous addition method and intermittent addition method) were adapted to decide the best desolvating agent. Obtained formulations were characterized and evaluated. The combination of acetonitrile and butanol as desolvating agent was giving good and promising results with good stability $-40.7 \mathrm{mV}$ and least mean particle diameter of $437.7 \mathrm{~nm}$. From the results it was concluded that intermittent addition was the better method than continuous addition method for preparing naproxen sodium nanoparticles. The drug release was sustained till $12 \mathrm{~h}$ with 96.92\% for nanoparticles prepared by intermittent addition method following zero order release with non- fickian diffusion mechanism.
\end{abstract}

Keywords: Desolvation; Bovine serum albumin; Cross linking agent; Butanol; SEM

\section{Introduction Definitions}

Nanoparticles (NPs) are defined as particulate dispersions or solid particles drug carrier that may or may not be biodegradable [1]. Nanoparticles are sub -nanosized colloidal structures composed of synthetic or semi-synthetic polymer, and the particle size ranges from 10-100 $\mathrm{nm}$ in diameter [19]. The drug is dissolved, entrapped, encapsulated or attached to a nanoparticle matrix. The term nanoparticle is a combined name for both nanospheres and nanocapsules [19].

\section{Nanocapsules}

Nanocapsules are vesicular system in which drug is essentially encapsulated with in the central volume surrounded by an embryonic polymeric sheath [1].

\section{Nanospheres}

Nanospheres are the spherical particulates with the size ranging between 10-200 $\mathrm{nm}$ in diameter [1]. Nanospheres contain drug embedded with the matrix 
(or) adsorbed on to the surface $[1,2]$.

The goal of any drug delivery system is to provide a therapeutic amount of drug to the proper site in the body [2]. The major focus on novel drug delivery systems during the past two decades is to improve the therapeutic efficacy and safety profile of drug substance [1]. Nanoparticles hold promise as drug delivery through various routes due to their good stability and easier manufacturing ability [18].

\section{Applications}

Different applications of nanoparticles are listed below: Brain targeted drug delivery [23]; delivery of anti-cancer drugs [2]; biomarker mapping [23]; probing of DNA structure [2]; treatment of autoimmune diseases [2]; used in ocular delivery [23]; used for directing anti-tubercular drugs [1]; used as adjuvant to vaccines [2]; and used as diagnostic agents [2].

\section{Advantages}

A nanoparticle offers numerous advantages in drug delivery system. These advantages include: Useful to diagnose various diseases [1]; enhanced stability of ingredients [22]; prolonged shelf life [22]; increased therapeutic efficacy; increased bioavailability [1]; nanoparticles shows better drug delivery as compare to other dosage forms and target to a particular cell or receptor [1]; more rapid onset of therapeutic action [22]; and nanoparticles can be administed by parenteral, oral or nasal routes [1].

\section{Disadvantages}

Nanoparticles have some disadvantages. These disadvantages include: It can lead to particle-particle aggregation due to their small size and large surface area [22]; small particle size results in limited drug loading [1]; polymeric nanoparticles are slowly biodegradable which causes systemic toxicity [1]; and susceptible to bursting and leakage of contents [22].

\section{Polymers for preparation of nano- particles}

The polymers used should be compatible within the body in terms of adaptability [23] and should be biodegradable and bio-compatible [23]. The polymers used for the preparation of nanoparticles are natural polymers and synthetic polymers.

\section{Natural polymers}

The most commonly used natural polymers in preparation of nanoparticles are: chitosan; gelatin; sodium alginate; and albumin.

\section{Synthetic polymers}

The most commonly used synthetic polymers in preparation of nanoparticles are [23]: Poly lactic acid; poly methyl methacrylate; polyacrylamide; and polyanhydride.

\section{Methods of preparation}

The various methods used for the preparation of nanoparticles are as follows [2]:

\section{Cross linking methods [2]}

Cross-linking of amphiphilic macromolecules; crosslinking in w/o emulsion; emulsion chemical dehydration; phase separation; and $\mathrm{pH}$ induced aggregation.

\section{Polymerization methods [2]}

Emulsion polymerization; dispersion polymerization; interfacial complexation; and interfacial condensation.

\section{Polymer precipitation methods [2]}

Salting-out method; nanoprecipitation; and solvent evaporation method.

Desolvation is the thermodynamically driven selfassembly process for polymeric materials [18]. This technique is mostly preferred for natural polymers. The addition of desolvating agents like butanol and acetonitrile coacervates the polymeric materials [18]. Desolvation is one of the common method reported for protein nanoparticle synthesis owing to its reproducibility, ease of preparation and simple process [1].

Naproxen is a member of the 2-arylpropionic acid family of NSAIDS having analgesic, anti-inflammatory and antipyretic activity (Fig. 1) [19]. Naproxen sodium is mainly used in the treatment of rheumatoid arthritis, bursitis, acute gout, osteoarthritis, juvenile arthritis, ankylosing spondylitis, headache, muscle aches, tendonitis, dental pain, and menstrual cramps [19]. It is also used for treating pain, swelling, and joint stiffness [19].

Naproxen is used to treat a variety of inflammatory conditions that are due to excessive inflammation, such 
<smiles>COc1ccc2cc(C(C)C(=O)O)ccc2c1</smiles>

Fig. 1 Structure of naproxen sodium.

as fever and pain (naproxen also has fever-reducing, or antipyretic, properties in addition to its antiinflammatory activity) [11].

The naproxen sodium salt is specifically indicated in the treatment of various types of acute and very high intensity pain because it induces a rapid and sustained remission [19].

On oral administration, naproxen mainly acts by inhibiting cyclo-oxygenase-1 (COX-1) and cyclooxygenase-2 (COX-2) receptors; naproxen induces an anti-inflammatory effect [19]. Cyclo-oxygenase is involved in the production of various chemicals in the body, some of which are known as prostaglandins [19]. Prostaglandins acts as signaling molecules in the body, inducing inflammation $[18,19]$. Inhibition of COX-1 causes severe gastro intestinal problems and inhibition of COX-2 causes severe cardiovascular problems [19]. In order to avoid these systemic side effects, it is better to develop novel drug delivery system for naproxen sodium to minimize the adverse effects and to reduce the frequency of administration [19]. To overcome these side effects, there is a need to employ novel drug delivery system for naproxen sodium [19].

Bovine serum albumin (BSA) is the most abundant globular protein in the plasma that is used in various biochemical applications [19]. Isolated BSA has been reported to be of drug and polymer profile [18]. BSA is a natural biodegradable polymer [20]. This protein is a commonly used protein due to its medicinal importance, low cost, water solubility, ready availability and stability [19]. Bovine serum albumin is a large globular protein with a good essential amino acid profile. Structurally, BSA is a single chain of 582 amino acids, non-glycoprotein, cross-linked with 17 cysteine residues [19].

The aim of the present study was to prepare naproxen sodium loaded BSA nanoparticles by desolvation technique using Butanol and combinations of acetonitrile and butanol as desolvating agents by continuous and intermittent addition methods. The effect of desolvating agent on the particle size was studied.

\section{Experimental Materials}

Drug: Naproxen sodium.

Polymer: BSA (Bovine serum albumin).

Desolvating agent: Butanol \& Acetonitrile.

Cross linking agent: Glutaraldehyde 25\%.

\section{Methods}

Naproxen sodium loaded BSA nanoparticles were prepared at various drug to polymer ratios, i.e. 1:1, $1: 2$ and $1: 3$, and by adapting the continuous addition method and the intermittent addition method. A total of eight formulations were prepared. Six formulations were prepared, i.e. F1, F2, F3, F4, F5 and F6 using butanol as desolvating agent, and two formulations prepared by using acetonitrile and butanol combinations as desolvating agent, i.e. F7 and F8.

\section{Desolvation technique}

The preparation of naproxen sodium loaded BSA nanoparticles was accomplished by the desolvation technique [5]. Desolvation is one of the common methods reported for protein nanoparticle synthesis owing to its reproducibility and ease of preparation. Two methodologies were followed for the addition of desolvating agent to the aqueous solution [11], including the continuous addition method and the intermittent addition method.

\section{Continuous addition method}

In continuous addition method, the desolvating agent was added at the rate of $1 \mathrm{~mL} / \mathrm{min}$ till the turbidity appears [18].

\section{Intermittent addition method}

In intermittent addition method, the desolvating agent was added at the rate of $1 \mathrm{~mL} / 5 \mathrm{~min}$ till the turbidity appears [18].

In desolvation technique, hydrophilic polymers are used to encapsulate both hydrophilic and hydrophobic drug entities [18].

Drug polymer solution was prepared and $\mathrm{pH}$ was adjusted to 7 (away from the iso-electric point) [18]. The desolvating agent was added under continuous mechanical stirring at $700 \mathrm{rpm}$ at room temperature [18]. A desolvating agent was added continuously and intermittently in to drug polymer solution. In 
continuous addition method, the desolvating agent was added at a rate of $1 \mathrm{~mL} / \mathrm{min}$. In intermittent addition method the desolvating agent was added at a rate of $1 \mathrm{~mL} / 5 \mathrm{~min}$ [18]. The appearance of turbidity was considered as the end point. Then a few millilitres of crosslinking agent (glutaraldehyde, 25\%) were added dropwise and kept for stirring for $8 \mathrm{~h}$. The solvent was removed by vacuum rotary evaporator. Free flowing nanoparticles were obtained and kept for air drying [19].

A total of six formulations were prepared by continuous and intermittent addition of butanol as desolvating agent and two formulations prepared using combinations of acetonitrile and butanol as desolvating agent.

\section{Evaluation and characterization of naproxen sodium nanoparticles}

The obtained formulations of naproxen nanoparticles prepared by desolvation technique were evaluated and characterized for the following parameters.

\section{Drug polymer compatibility study using Fourier transforms infrared spectroscopy (FTIR)}

Compatibility studies were performed using FTIR spectrophotometer. The infrared (IR) spectrum of the formulations were studied [20]. The FTIR spectra acquired were taken for the dried samples. Naproxen sodium and BSA prepared nanoparticles were mixed separately with finely powdered potassium bromide IR [9]. Grind the mixture thoroughly, spread it uniformly in a suitable die and compressed under vacuum pressure of about 8000 metric tons of pressure in a hydraulic press, and the pellets were scanned over a wave number range of 4000 to $400 \mathrm{~cm}^{-1}$ in a FTIR instrument [9]. Mount the resultant disc in a suitable holder in the spectrophotometer [20].

\section{Surface morphology of nanoparticles using scanning electron microscope (SEM)}

The prepared nanoparticles were dispersed in deionised water and sonicated for $30 \mathrm{~min}$ [18]. And then a drop of the diluted nanoparticles suspension was directly deposited on a polished aluminium sample holder [18]. The samples were dried in the vacuum. The nanoparticles were viewed at an accelerating voltage of 15-20 kV. Then, it was scanned under SEM for morphology [18].

\section{Particle size analysis}

The mean particle size of the nanoparticles was determined by photon correlation spectroscopy (PCS) with a Malvern Zetasizer Nano ZS (Malvern Instruments, Malvern, UK). Measurements were realized in triplicate at a $90^{\circ}$ angle at $25^{\circ} \mathrm{C}$ under suitable dilution conditions. The prepared nanoparticles were dispersed in deionised water and sonicated for few minutes [18]. The resultant dispersion was diluted and observed for particle size [18].

\section{Zeta potential}

The zeta potential (surface charge) which indicates the physical stability of the nanoparticles was determined by electrophoretic mobility [18]. Samples were prepared by diluting with water and corresponding zeta potential were measured using Malvern Zetasizer Nano ZS (Malvern Instruments, Malvern, UK) [1].

\section{Percentage yield}

Also called as product yield. Nanoparticles are dried at room temperature were weighed [1].

The yield of nanoparticles was calculated using the following formula:

Percentage yield [9] $=$ (Amount of obtained nanoparticles/Theoretical amount) $\times 100$.

\section{Drug content}

The nanoparticle formulations were examined for drug content. Prepared nanoparticles were dissolved in a solvent in which the drug was completely soluble [18]. It was subjected to stirring at $700 \mathrm{rpm}$ for $3 \mathrm{~h}$ by using magnetic stirrer [18]. The resultant solution was filtered and the amount of the drug in the filtrate was estimated after suitable dilution by UV spectrophotometer at $271 \mathrm{~nm}$ [17].

\section{Entrapment efficiency}

Entrapment efficiency indicates the amount of drug encapsulated in the formulation [19].

Entrapment efficiency is conducted by taking prepared nanoparticles suspension in equivalent quantity of $\mathrm{pH} 7.4$ phosphate buffer [18]. The nanoparticle suspension was ultra-centrifuged at $17000 \mathrm{rpm}$ and temperature of $-4{ }^{\circ} \mathrm{C}$ for $40 \mathrm{~min}$ [6]. 
The amount of drug present in the clear supernatant after centrifugation was determined by UV spectrophotometer at a wavelength of $271 \mathrm{~nm}$ [18].

The percentage entrapment efficiency was calculated with the following formula:

Entrapment efficiency [9] $=$ (Amount of drug encapsulated in the formulation/Total amount of drug in the formulation) $\times 100$.

\section{Loading capacity}

It indicates the capacity of the polymer to load a drug [18].

Loading capacity was calculated from the following formula: Loading capacity [9] = (Total amount of the drug I amount of free drug concentration / Nanoparticles' weight) $\times 100$.

\section{In vitro drug release study}

In vitro drug release studies were performed in an orbital shaker [18]. Drug release from polymeric nanoparticles was determined as follows. A known amount of nanoparticles was transferred to a conical flasks with $50 \mathrm{~mL}$ of $\mathrm{pH} 7.4$ phosphate buffer. They were kept in orbital shaker at a temperature of $37(+/-) 0.5{ }^{\circ} \mathrm{C}$ and rotation speed of $100 \mathrm{rpm}$ for $12 \mathrm{~h}$ [18]. The samples were withdrawn at predefined time intervals and replaced with same amount of fresh buffer. The samples were further analyzed using UV spectrophotometer at a wavelength of $271 \mathrm{~nm}$ [19].

\section{Results and Discussion}

The obtained formulations were characterized and evaluated for the above parameters and the results are discussed as follows:

\section{Evaluation and characterization of naproxen sodium loaded BSA nano- particles by desolvation technique}

Naproxen sodium loaded BSA nanoparticles were prepared at various drug-polymer ratios i.e. (1:1, $1: 2$ and $1: 3$ ) and two methods, i.e. continuous and intermittent addition methods were adapted for the preparation of polymeric nanoparticles.

\section{Evaluation and characterization of naproxen nanoparticles Scanning electron microscopy}

The naproxen nanoparticles prepared by desolvation technique. The optimized formulations F2 and F8 were characterized for surface morphology using scanning electron microscopy (S-3700N, Hitachi, Mumbai). From the resultant images, it was confirmed that the particles were found to be spherical in shape and having the size within nanometer range (Fig. 2 and 3 ).

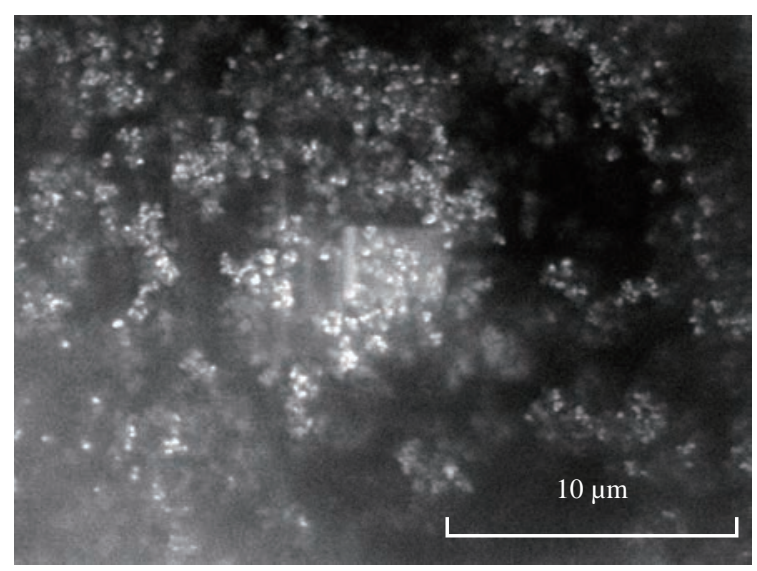

Fig. 2 SEM image of F2 formulation of naproxen loaded BSA nanoparticles by using butanol as desolvating agent.

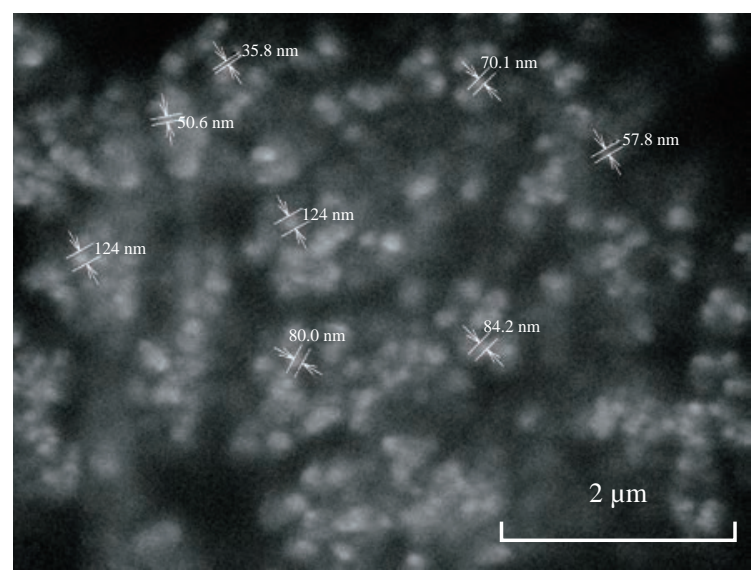

Fig. 3 SEM image of F8 formulation of naproxen loaded BSA nanoparticles by using combinations of acetonitrile and butanol as desolvating agent.

\section{Fourier transforms infrared spectroscopy (FTIR)}

Compatibility studies were performed using FTIR spectrophotometer [20]. In the FTIR spectrum $\mathrm{C}-\mathrm{H}$ stretching vibration at $3153-3059 \mathrm{~cm}^{-1}, \mathrm{C}-\mathrm{O}$ stretching vibrations at $1300-1000 \mathrm{~cm}^{-1}$, aromatic $\mathrm{C}=\mathrm{C}$ stretching vibration at $1631 \mathrm{~cm}^{-1}$ and $\mathrm{CH}_{3}$ bending vibration at $1363 \mathrm{~cm}^{-1}$, indicating the significant peaks of naproxen sodium [20], thus indicating no drug-polymer interaction [20] (Fig. 4).

\section{Mean particle size}

The mean particle diameter of the optimized 


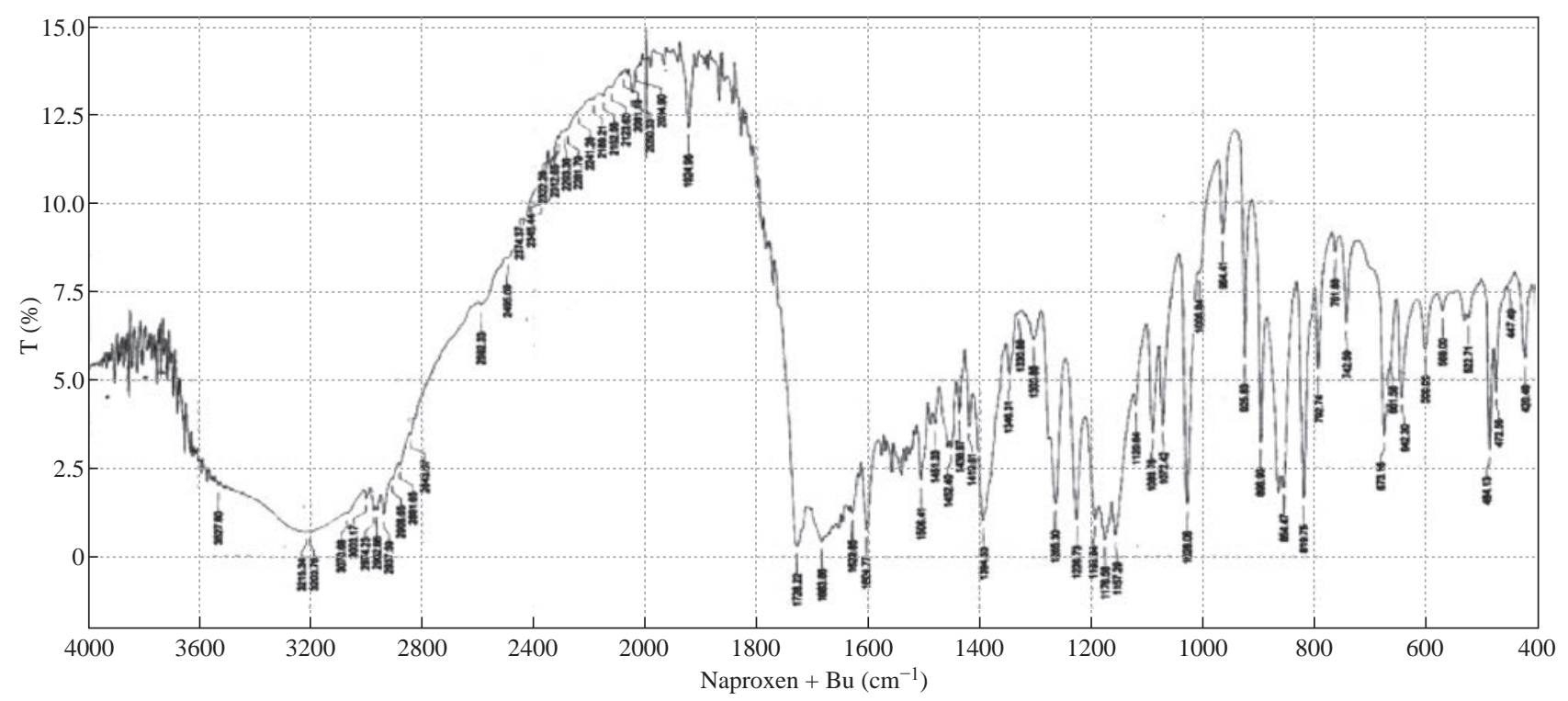

Fig. 4 FTIR spectra of F2 formulation of Naproxen loaded BSA nanoparticles by addition of butanol as desolvating agent.

formulations was determined by photon correlation spectroscopy (PCS) with a Malvern Zetasizer Nano ZS (Malvern Instruments, Malvern, UK). The formulations were within nano range. The formulation, i.e. F2 prepared using butanol as desolvating agent showed mean particle size of $385.1 \mathrm{~nm}$, and the formation F8 prepared using combinations of acetonitrile and butanol as desolvating agent showed mean particle size of $437.7 \mathrm{~nm}$.

\section{Zeta potential}

Zeta potential of the optimized formulations was determined for zeta potential value in order to reveal the stability of the formulation using Malvern Zetasizer (Malvern Instruments, Malvern, UK). F2 formulation was showing zeta potential of $-16.5 \mathrm{mV}$ and $\mathrm{F} 8$ was showing zeta potential of $-40.7 \mathrm{mV}$. F8 formulation was showing good stability.

\section{Product yield}

The dried nanoparticles were calculated for product yield. A product yield of all prepared formulations was compared using butanol as desolvating agent. They were found to be $78.12 \%, 77.45 \%, 54.52 \%, 57.78 \%$, $74.37 \%$ and $80.35 \%$ (Fig. 5).

The product yield of the two formulations prepared using combinations of acetonitrile and butanol as desolvating agent was found to be $79.42 \%$ and $80.64 \%$.

\section{Drug content}

Drug content of all the prepared formulations was compared. They were found to be $95.03 \%, 86.98 \%$, $70.81 \%, 82.34 \%, 88.37 \%$ and $93.02 \%$ (Fig. 6 ).

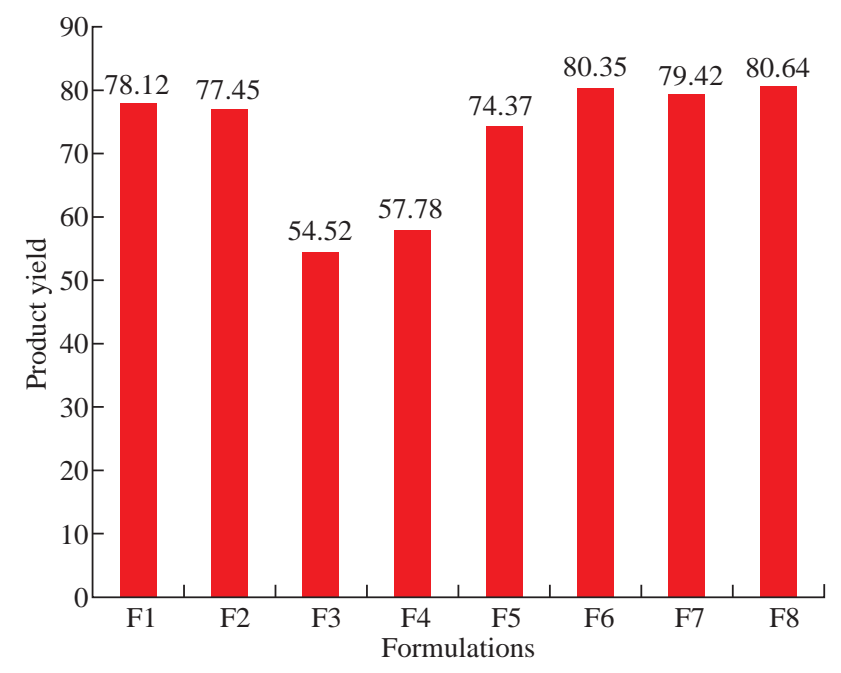

Fig. 5 Product yield of nanoparticles.

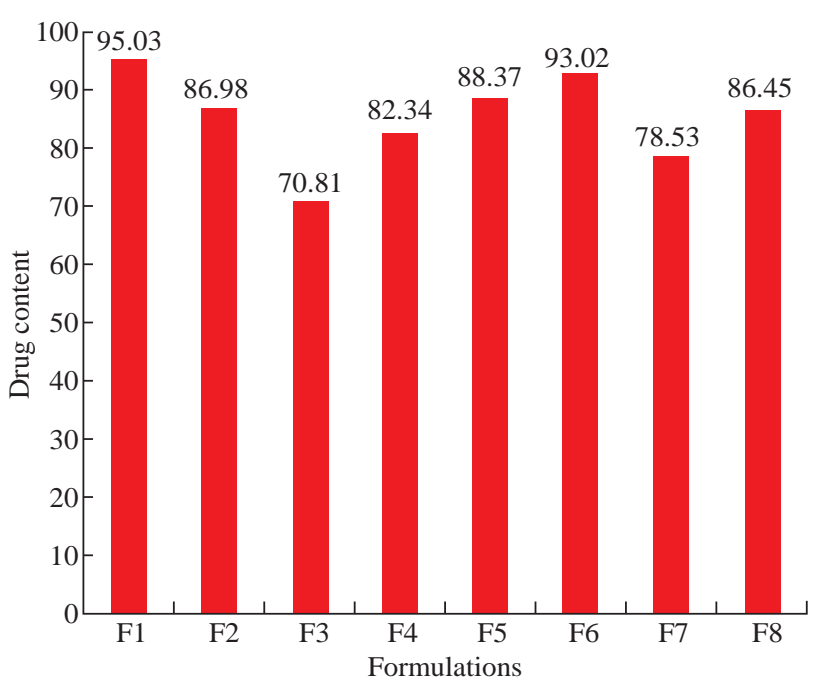

Fig. 6 Drug content of nanoparticles. 
Drug content of the two formulations prepared using combinations of acetonitrile and butanol as desolvating agent was found to be $78.53 \%$ and $86.45 \%$ (Fig. 6).

\section{Entrapment efficiency}

Entrapment efficiencies of all prepared formulations were compared. They were found to be $19.04 \%$, $76.01 \%, 33.59,20.88 \%, 59.14 \%$ and $19.35 \%$ (Fig. 7).

Entrapment efficiency of the two formulations prepared using combinations of acetonitrile and butanol as desolvating agent was found to be $70.73 \%$ and $76.72 \%$ (Fig. 7 ).

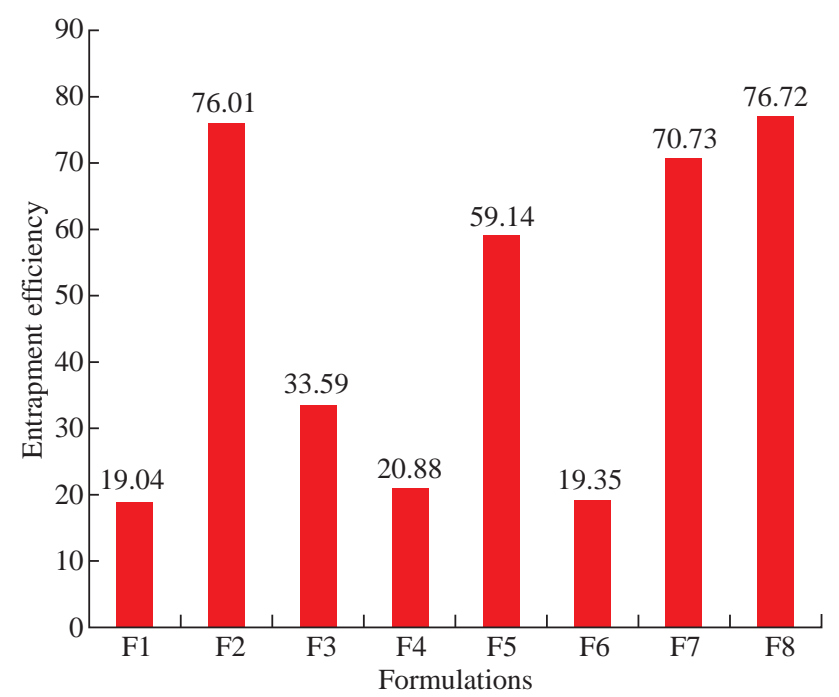

Fig. 7 Entrapment efficiency of nanoparticles.

\section{Loading capacity}

Loading capacities were found to be $6.4,28.2$, 4.3, 3.3, 9.7 and 3.6 for formulations prepared using butanol as desolvating agent.

Loading capacity of the two formulations prepared using combinations of acetonitrile and butanol as desolvating agent was found to be $22 \%$ and $26.7 \%$.

\section{In-vitro drug release studies}

All the prepared formulations were evaluated for Invitro drug release study. From the results it was found to be $98.05 \%, 86.93 \%, 97.57 \%, 90.86 \%, 89.02 \%$ and $99.30 \%$ within a period of $12 \mathrm{~h}$ (Fig. 8).

Drug release of the two formulations prepared using combinations of acetonitrile and butanol as desolvating agent was found to be $71.58 \%$ and $96.92 \%$ (Fig. 8).

\section{Kinetics of drug release}

Different plots (zero order, first order, Higuchi and Korsmeyer-Peppas plots) were drawn for the optimized formulations (F2 and F8), in order to know the release kinetics of the drug and also drug release mechanism.

According to the kinetic plots, the optimized formulations, i.e. F2 and F8 (Fig. 9 and 10) followed

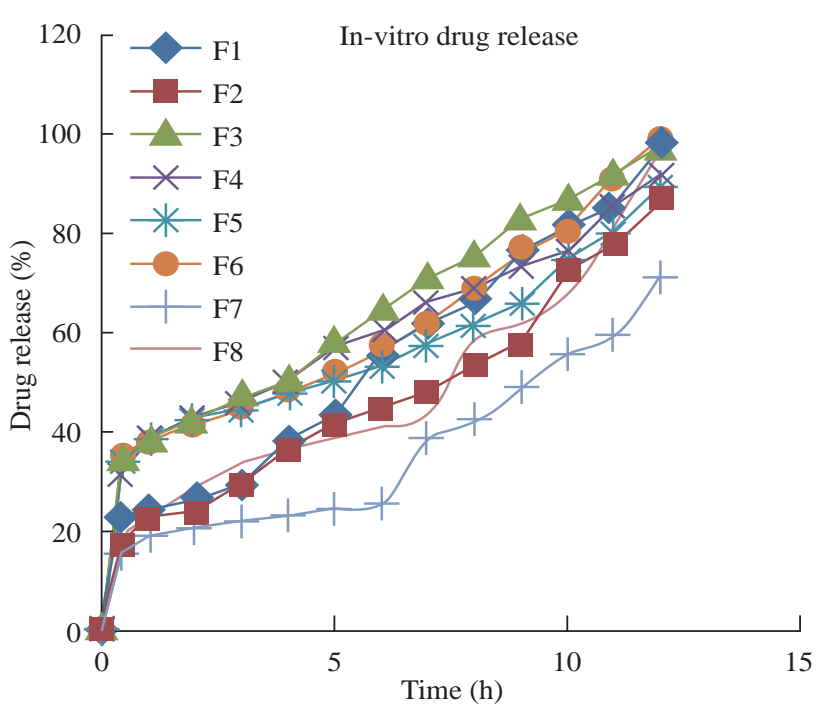

Fig. 8 In vitro drug release pattern of nanoparticles.

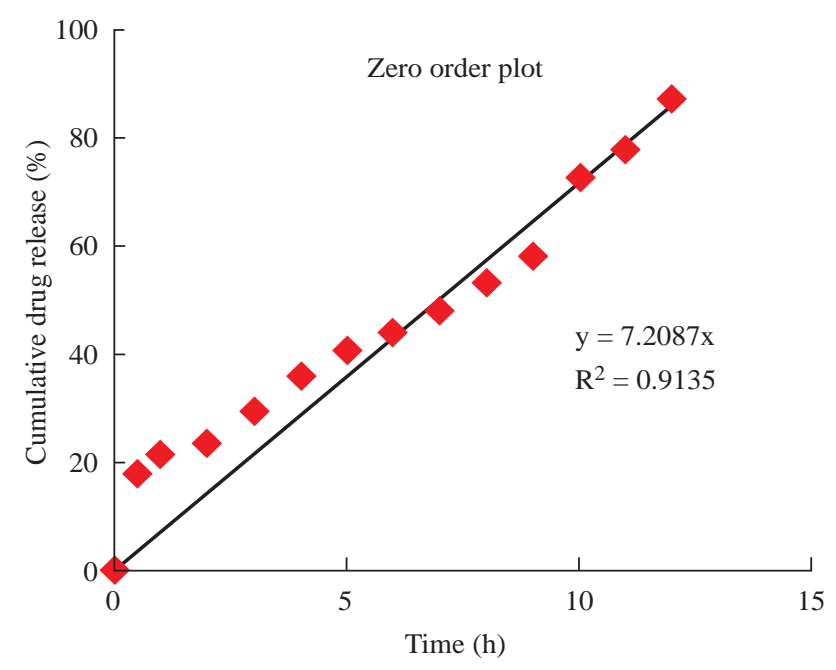

Fig. 9 Zero order release for optimized formulation F2.

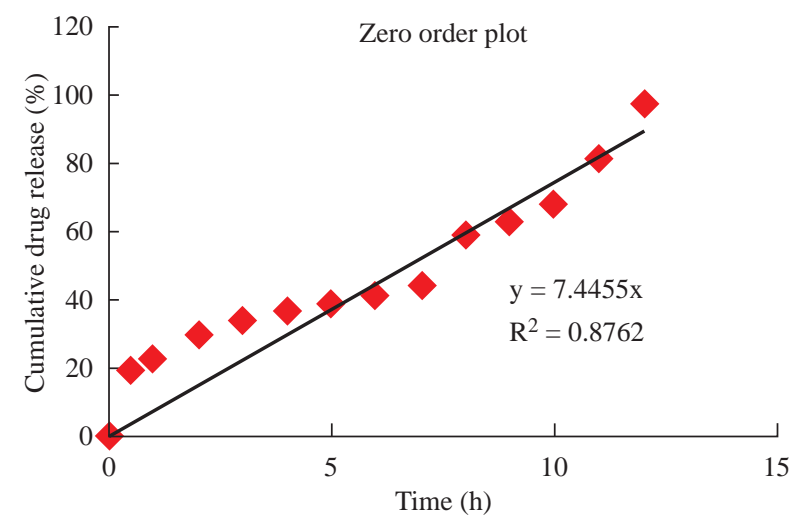

Fig. 10 Zero order release for optimized formulation F8. 
zero order release with non-fickian diffusion mechanism as shown in Table 1.

Table 1 Kinetic release data for optimized formulation F2 and F8

\begin{tabular}{ccccc}
\hline Formulation & $\begin{array}{c}\text { Zero order } \\
\left(\mathrm{R}^{2}\right)\end{array}$ & $\begin{array}{c}\text { First order } \\
\left(\mathrm{R}^{2}\right)\end{array}$ & $\begin{array}{c}\text { Higuchi plot } \\
\left(\mathrm{R}^{2}\right)\end{array}$ & Peppas plot (n) \\
\hline F2 & 0.913 & 0.871 & 0.838 & 0.693 \\
F8 & 0.876 & 0.786 & 0.838 & 0.618 \\
\hline
\end{tabular}

\section{Discussion}

Naproxen sodium is a non-steroidal antiinflammatory drug. Naproxen has fever-reducing, or antipyretic, properties in addition to its antiinflammatory activity [19]. Naproxen sodium has been used as the first line drug in the treatment of rheumatoid arthritis, osteoarthritis and ankylosing spondylitis [18].

On prolonged usage, it causes severe gastrointestinal (GI) complications and peptic ulcers [20]. In order to reduce the dosing frequency and adverse effects of the drug, novel approach has been implemented in this study to prepare naproxen nanoparticles [18].

According to the American Gastroenterological Association, more deaths are due to NSAIDS complications than from AIDS and cancer each year. In many cases, the first evidence of NSAIDS toxicity is a GI complication [19]. In order to avoid these severe adverse effects, there is a need to develop novel drug delivery system for naproxen sodium [18].

In the present study, naproxen sodium loaded BSA nanoparticles were prepared by desolvation technique using two desolvating agents, i.e. butanol and combinations of acetonitrile and butanol as desolvating agent. Naproxen sodium loaded BSA nanoparticles were prepared by altering drug to polymer ratio $(1: 1$, $1: 2$ and 1:3) and two methods, i.e., the continuous and the intermittent addition methods were adapted for the preparation of polymeric nanoparticles. Six formulations were prepared with each desolvating agent, i.e. F1, F2, F3, F4, F5 and F6 using butanol as desolvating agent and two formulations prepared by using combinations of acetonitrile and butanol as desolvating agent, i.e. F7 and F8.

Acetonitrile is a polar aprotic solvent. Consequently, they are not capable of hydrogen bonding and aprotic solvents are generally incompatible with strong bases [23], while butanol is a polar protic solvent that has a hydrogen atom bound to oxygen and nitrogen as in an amine group. Polar protic solvents are very effective at stabilizing ions and are capable of hydrogen bonding [24].

Among six formulations of butanol, F2 was considered as the best formulation because of its least mean particle size diameter of $385.1 \mathrm{~nm}$ with zeta potential of $-16.5 \mathrm{mV}$. The percentage yield, drug content, entrapment efficiency and loading capacity of the best formulation were found to be $77.45 \%, 86.98 \%$, $76.01 \%$ and $28.2 \%$ respectively, and was sustained till $12 \mathrm{~h}$ with $86.93 \%$ of drug release following zero order release with non-fickian diffusion mechanism.

F7 and F8 formulations were prepared by a combination of acetonitrile and butanol as desolvating agents and were evaluated for all the parameters. F7 was prepared by continuous addition method whereas F8 was prepared by intermittent addition method. F8 formulation was prepared by using the combinations of acetonitrile and butanol as desolvating agent and was considered the best formulation because of its least mean particle size diameter of $437.7 \mathrm{~nm}$ with zeta potential of $-40.7 \mathrm{mV}$. The percentage yield, drug content, entrapment efficiency and loading capacity of the best formulation was found to be $80.64 \%, 86.45 \%$, $76.72 \%$ and $26.7 \%$, respectively, and was sustained till $12 \mathrm{~h}$ with $96.92 \%$ of drug release following zero order release with non-fickian diffusion mechanism.

In intermittent addition method, the drug release was $96.92 \%$, which may be because of the smaller particle size. Smaller particle size diameter provides maximum surface area, the drug release was found to be maximum in a period of $12 \mathrm{~h}$. In continuous addition method, the drug release was $71.58 \%$. Sustained release nature was because of the large diameter of the particle [19].

Intermittent addition method was considered as a more suitable method than continuous addition method, because intermittent addition method involved more time for the desolvation process to occur and also resulted in particles in nanosize compared to continuous addition method.

Intermittent addition method was considered as the excellent method because less volume of desolvating agent was consumed when compared to continuous addition method [19]. 
Comparing the continuous and the intermittent addition methods, we found that the intermittent addition method was giving good and promising results. Butanol formulation F2 was giving good and promising results. The least mean particle size diameter of $385.1 \mathrm{~nm}$ was obtained with zeta potential of -16.5 $\mathrm{mV}$.

Naproxen nanoparticles prepared by using acetonitrile and butanol combination as desolvating agent was considered as the best and most appropriate desolvating agent for the preparation of naproxen nanoparticles because of its good stability as of -40.7 $\mathrm{mV}$, drug content of $86.45 \%$, entrapment efficiency of $76.72 \%$, loading capacity of $26.7 \%$, mean particle size diameter as of $437.7 \mathrm{~nm}$, and in vitro drug release data showed $96.92 \%$ of drug release rate profile which sustained up to $12 \mathrm{~h}$.

\section{Conclusions}

Naproxen sodium is an anti-inflammatory agent with analgesic and antipyretic properties [18]. It is used in the treatment of rheumatoid arthritis and other musculoskeletal disorders [11].

In the present study, efforts have been made to prepare naproxen nanoparticles by desolvation technique using butanol and combinations of acetonitrile and butanol as desolvating agent. Formulations were evaluated for product yield, drug content, entrapment efficiency, and loading capacity. The mean particle diameter and zeta potential values were compared. Based on the results, the formulation F8 showed the smallest particle size and a greater stability. In comparison to all the parameters, the combinations of acetonitrile and butanol was considered as good desolvating agent for the preparation of naproxen nanoparticles. The combination of acetonitrile and butanol was giving good and favourable results. Intermittent addition method was considered as the best and appropriate method for the preparation of naproxen loaded BSA nanoparticles.

\section{Acknowledgments}

The authors sincerely thank Dr. M. Sumakanth, the Principal, RBVRR Women's College of Pharmacy for providing access to the library and databases. We also thank Mrs. Suvarna and Mrs. Sumalatha, for providing technical assistance.

\section{Conflict of Interests}

The authors declare that no competing interest exists.

\section{References}

[1] N.K. Jain, Advances in controlled and novel drug delivery. CBS Publishers and Distributers, New Delhi, 2001: 408.

[2] S.P. Vyas, R.K. Khar, Targeted \& Controlled Drug Delivey, Novel Carrier Systems. CBS Publication, 2002: 331-381.

[3] C. Weber, C. Coester, J. Kreuter, et al., Desolvation process and surface characterisation of protein nanoparticles. International Journal of Pharmaceutics, 2000, 194(1): 91-102.

[4] D.M. Brahmankar, S.B. Jaiswal, Biopharmaceutics \& Pharmaceutics, $1^{\text {st }}$ edition. Vallabhprakashan Publications, 1995: 162-163, 165, 335.

[5] A.K. Sailaja, P. Amareshwar, and P. Chakravarty, Different techniques used for the preparation of nanoparticles using natural polymers and their applications. International Journal of Pharmacy and Pharmaceutical Sciences, 2011, 3(2): 45A-50A.

[6] M. Rahimnejad, M. Jahanshahi, and G.D. Najafpour, Production of biological nanoparticles from bovin serum albumin for drug delivery. African Journal of Biotechnology, 2006, 5(20): 1918-1923.

[7] M. Abhilash, Docking methods. International Journal of Pharma and Bio Sciences, 2010, 1(1): 1-12.

[8] V.J.M. Raj, Y. Chen, Nanoparticles - A review. Tropical Journal of Pharmaceutical Research, 2006, 5: 561.

[9] A.K. Sailaja, A comparative study of aspirin loaded bovine serum albumin nanoparticles prepared by desolvation technique using various desolvating agents. Nano Biomedicine and Engineering, 2017, 9(2): 143-151.

[10] A.K. Sailaja, A. Sarita, Preparation and characterization of Aspirin loaded ethyl cellulose nanoparticles by solvent evaporation technique. World Journal of Pharmacy and Pharmaceutical Sciences, 2014, 3(6): 1781-1793.

[11] J. Banu, A.K. Sailaja, Preparation and characterize polymeric nanoparticles of naproxen by emulsion interfacial reaction method \& desolvation techniques. Journal of Bionanoscience, 2017, 12(2): 159-169.

[12] K. Sailaja, Preparation of diclofenac nanoparticles by desolvation technique using acetone as desolvating agent. Indian Journal of Novel Drug Delivery, 2016, 8: 42-45.

[13] N. Begum, K.A. Sailaja, Effect of formulation variables on the Preparation of Ibuprofen loaded polymeric nanoparticles. Pharmaceutical Nanotechnology, 2015: 111-121.

[14] S. Tyagi, V.K. Pandey, Nanoparticles: An overview of preparation. Journal of Pharmaceutics and Nanotechnology JPN, 2016, 4 (2).

[15] N. Jawahar, S.N. Meyyanathan, Polymeric nanoparticles for drug delivery and targeting: A comprehensive review. International Journal of Health and Allied Sciences, 2012, 1: 217-223.

[16] K. Adibkia, Y. Javadzadeh, S. Dastmalchi, et al., Naproxen-eudragit RS100 nanoparticles: preparation and physicochemical characterization. Colloids and Surfaces B: Biointerfaces, 2011: 155-159.

[17] A.K. Sailaja, P Amareshwar, Preparation of BSA 
nanoparticles by desolvation technique using acetone as desolvating agent. International journal of Pharmaceutical Science and Nanotechnology, 2012, 5: 1643-1647.

[18] A.K. Sailaja, M. Nandini, Effect of various desolvating agents in the formulation of naproxen loaded BSA nanoparticles. Journal of Bionanoscience, 2017, 11(6): 497-503.

[19] A.K. Sailaja, M. Shreya, Preparation and characterization of naproxen loaded niosomes by ether injection method. Nano Biomedicine and Engineering, 2018, 10(2): 174180.

[20] G. Archana, Formulation and evaluation of naproxen sodium loaded bovin serum albumin nanoparticles by desolvation technique. Asian Journal of Pharmaceutical Technology \& Innovation, 2015, 3(14); 23-33.

[21] B. Nagavarma, V.N. Hemant, K.S. Yadav, et al., Different techniques for preparation of polymeric nanoparticles A review. Asian Journal of Pharmaceutical and Clinical Research, 2012, 5(Suppl 3): 16-23.
[22] M.A. Godwin, K.M. Shri, Nanoparticles and their applications - A mini review. International Journal of Research in Engineering and Bioscience, 2015, 3(5): 1129.

[23] U.S. National Library of Medicine, National Center for Biotechnology Information Compound summary: Acetonitrile. <https://pubchem.ncbi.nlm.nih.gov/ compound/acetonitrile>, retrieved on Jan. 2, 2019.

[24] U.S. National Library of Medicine, National Center for Biotechnology Information Compound summary: 1-Butanol. <https://pubchem.ncbi.nlm.nih.gov/ compound/1-butanol>, retrieved on Jan. 1, 2019.

Copyright $\odot$ Huda Syed, Abbaraju Krishna Sailaja. This is an open-access article distributed under the terms of the Creative Commons Attribution License, which permits unrestricted use, distribution, and reproduction in any medium, provided the original author and source are credited. 